\title{
Corps médical et industrie
}

Dans le monde anglo-saxon, les membres du corps médical et de l’industrie sont désignés sous le terme de «uneasy bedfellows» (partenaires aux relations complexes). Par le biais du Bulletin des médecins suisses, le Comité central de la FMH a publié en 1990 des recommandations sur le problème et le code de déontologie de la FMH de 1997 en a énoncé quelques principes essentiels.

D'entente avec le Comité central de la FMH, un groupe de travail de la commission d'assurancequalité de la Société suisse de gynécologie et obstétrique s'est lui aussi penché sur la question. Nous lui en sommes reconnaissants.

Les premières recommandations, publiées ci-après, se limitent au sujet le plus brûlant. Le droit pénal suisse révisé exige en effet un examen rapide et critique des relations entre l'industrie et le service public et une transparence accrue en ce domaine.

Nous remercions tout particulièrement M. le Prof. Urs Haller, président de la commission assurancequalité de la Société suisse de gynécologie et obstétrique, ainsi que M. le Dr Gero Drack, président de la sous-commission "Corps médical et industrie», pour leur engagement sans faille lors de l'élaboration de ces très utiles et combien nécessaires informations.

Dr H. H. Brunner, président de la FMH 